\title{
Predicting postnatal mental disorder with a screening questionnaire: a prospective cohort study from Zimbabwe
}

\author{
Sekai Nhiwatiwa, Vikram Patel, Wilson Acuda
}

\begin{abstract}
Background-Postnatal mental disorders are common causes of morbidity but are rarely diagnosed or treated in busy primary care clinics in developing countries. Objective-To determine whether a brief psychiatric screening questionnaire used in the 8th month of pregnancy can predict postnatal mental disorder.
\end{abstract}

Study design-Prospective cohort study. Site-A peri-urban settlement in Zimbabwe.

Population-500 women in the 8th month of pregnancy identified by traditional birth attendants and primary care clinics. Sample-"High risk" cohort consisted of all women who scored 8 or more on the Shona Symptom Questionnaire (SSQ), an indigenous psychiatric questionnaire $(n=95)$. Low risk cohort consisted of 105 women randomly selected from the remainder of the sampling frame.

Outcome measure-Revised Clinical Interview Schedule at six to eight weeks postpartum; scores of 14 or more indicate psychiatric caseness.

Results-The prevalence of postnatal mental illness was $16 \%$. Odds ratios $(95 \%$ confidence intervals) for high risk women becoming cases in the postnatal period were 10.6, 4.8, 23.9, $\mathrm{p}<0.0001$ after adjustment for age, marital status, and occupation.

Conclusions-A brief method of detecting women at high risk of developing a postnatal mental disorder can be used with reasonable accuracy in the 8 th month of pregnancy. Further research is needed to determine whether interventions applied to this high risk group can reduce their postnatal morbidity.

Psychiatry, University of Zimbabwe Medical

School, Harare,

Zimbabwe

S Nhiwatiwa

W Acuda

Section of

Epidemiology,

Institute of Psychiatry,

London

V Patel

Correspondence to:

Dr V Patel, Institute of

Psychiatry and Human

Behaviour, Altinho, Panjim,

Goa 403001, India.

Accepted for publication 5 June 1997 to be comprehensive, awareness, diagnosis, and management of postnatal mental disorders is rare. Epidemiological evidence from industrialised societies and anecdotal evidence from Africa show that untreated postnatal mental illness can last up to a year. These disorders can be effectively managed with psychological and antidepressant treatment. ${ }^{3}$ There is a paucity of systematic research work on puerperal mental disorders in Africa. Assael et al reported from a rural study in Uganda that $24 \%$ of pregnant women had a psychiatric disorder and $16 \%$ had a depressive syndrome after delivery. ${ }^{4}$ Cox studied women attending an antenatal clinic at a health centre in Uganda and found that 30\% had psychiatric morbidity in the antenatal period and among those followed up into puerperium $10 \%$ suffered a postnatal depression. ${ }^{56}$ Aderibibge et al in a study of women attending antenatal care at the University Teaching Hospital, Ibadan, Nigeria found $30 \%$ of women had a prenatal psychiatric disorder and $14 \%$ had postnatal psychiatric disorder. $^{7}$

Research for accurate predictors of postnatal mental illness can take two directions: firstly, there is growing evidence that postnatal disorders are often associated with psychological morbidity in the late antenatal period and early postnatal period. ${ }^{2}$ Secondly, putative risk factors for postnatal depression, such as a complicated delivery and previous history of depression, may be used as predictors. ${ }^{8}$ The key advantage of identifying such predictors is that there is a potential to reduce the morbidity of postnatal disorders by targeting interventions at mothers at high risk. For example, there is evidence that preventive strategies such as inviting mothers at high risk to support groups and closely following up women with maternity blues or those who score high on psychological measures in the late antenatal or early postnatal period are both acceptable to mothers and lead to reduced morbidity in the postnatal period. $^{9}{ }^{10}$

The hypothesis of the study described in this paper was that psychological disorder in the 8th month of pregnancy was associated with postnatal mental illness. The aim of this study was to determine whether this morbidity as detected by a simple screening questionnaire of psychiatric symptoms in the 8th month of pregnancy predicted a postnatal mental disorder. The rationale for the study was that a brief screening method could be easily integrated into the routine work of busy antenatal clinics and community midwives working in a comparatively impoverished area of a low income country.

\section{Methods}

A prospective cohort study was carried out in Epworth, which is a peri-urban area on the outskirts of the city of Harare, Zimbabwe. It is 
Table 1 Significant differences between high and low risk groups

\begin{tabular}{|c|c|c|c|c|}
\hline Variable & $\begin{array}{l}\text { Proportion of high } \\
\text { risk (\%) }\end{array}$ & Proportion of low risk (\%) & $\begin{array}{l}\text { Odds ratios }(95 \% \\
\text { CI) }\end{array}$ & Odds ratios adjusted for age \\
\hline $\begin{array}{l}\text { Number of children } \\
3 \text { or more }\end{array}$ & 32 & 15 & $\begin{array}{l}2.6(1.3,5.1) \\
p=0.006\end{array}$ & $\begin{array}{l}2.6(1.1,6.3) \\
p=0.03\end{array}$ \\
\hline $\begin{array}{l}\text { Number of miscarriages } \\
1 \text { or more }\end{array}$ & 22 & 11 & $\begin{array}{l}2.4(1.1,5.1) \\
p=0.02\end{array}$ & $\begin{array}{l}2(0.9,4.5) \\
p=0.08\end{array}$ \\
\hline $\begin{array}{l}\text { Relationship with husband } \\
\text { Unhappy }\end{array}$ & 10 & 3 & $\begin{array}{l}3.7(0.99,14.3) \\
p=0.05\end{array}$ & $\begin{array}{l}4.8(1.2,18.6) \\
p=0.02\end{array}$ \\
\hline $\begin{array}{l}\text { Mothers residence } \\
\text { In rural areas }\end{array}$ & 73 & 52 & $\begin{array}{l}2.5(1.2,4.9) \\
\mathrm{p}=0.01\end{array}$ & $\begin{array}{l}2.3(1.1,4.6) \\
\mathrm{p}=0.01\end{array}$ \\
\hline Current medical problems & 79 & 44 & $\begin{array}{l}4.7(2.5,8.8) \\
\mathrm{p}<0.001\end{array}$ & $\begin{array}{l}4.8(2.6,9.1) \\
\mathrm{p}<0.001\end{array}$ \\
\hline
\end{tabular}

an impoverished area where antenatal health services consist of two primary care clinics and traditional birth attendants. There were no full time doctors in Epworth at the time of this study.

The population was consecutive women $(n=500)$ in the eighth month of pregnancy identified either at the antenatal clinics of primary health clinics or by TBAs in the community. Consent was obtained from each woman before recruitment for the study. The entire population was screened using the Shona Symptom Questionnaire (SSQ), a 14 item indigenous measure of non-psychotic psychological morbidity. The validity data of this questionnaire show that a cut off score of 7 of 8 has the optimal balance of sensitivity and specificity for case detection. ${ }^{11}$ Piloting of the SSQ with a sample of community based pregnant women was conducted simultaneously with piloting of the outcome measure, the Revised Clinical Interview Schedule (see below). The SSQ is principally based on local idioms and was found to be acceptable to women; furthermore, because of its emphasis on cognitive symptoms (12 out of 14), the risk of confounding caused by the somatic experiences associated with pregnancy was considered by the interviewers (village health workers) to be minimal. Based on the SSQ score, each woman was classified into either of two cohorts - that is, women who had psychological morbidity (score of 8 or more and referred to as the "high risk" group) and women who did not (score of 7 or less and referred to as the "low risk" group). Both cohorts were interviewed with a questionnaire eliciting sociodemographic data (appendix). This screening procedure was conducted by village health workers who were in contact with all pregnant women in the area. All women gave written informed consent to participate in the study. The sample size was based on a power calculation that estimated the odds of the high risk group developing the postnatal depression at 4 (with $5 \%$ expected prevalence in the low risk group, $80 \%$ power, $90 \%$ confidence).

All women in the "high risk" group and a random sample of the "low risk" group were reviewed at six to eight weeks postpartum using the Shona version of the Revised Clinical Interview Schedule (RCIS). The RCIS is a standardised psychiatric interview for use in community and primary care studies. ${ }^{12}$ It consists of 14 domains that reflect non-psychotic morbidity, for example, somatic symptoms, fatigue, concentration, sleep, irritability, worry about physical health, depression, depressive ideas, worry, anxiety, panic, phobias, obsessions, and compulsions. Its total score can range from 0 to 57 ; the score is a measure of the degree of non-psychotic psychiatric morbidity. The translation, reliability, and use of the Shona version of the interview is described elsewhere. ${ }^{13}$ A cut off score of $13 / 14$ was determined as being sensitive for identifying cases on the basis of a pilot study where the criterion of caseness was psychiatric evaluation by the first author. Thus, women who scored 14 or more were classified as "cases" and the remainder as "non-cases". Six to eight weeks postpartum was chosen as the follow up interview period because the onset of postnatal depression is characteristically associated with this period. Data on the obstetric history of the recent pregnancy was also collected at this stage. The follow up interviews were conducted by the first author who was blind to the recruitment SSQ scores. Further interviews were not possible because of time and financial constraints. Only statistically significant results are presented. Percentage figures are rounded off to the nearest integer.

\section{Results}

Of the population of 500, none of the women refused to take part in the study. Of the population, 95 women scored 8 or more on the SSQ thus giving a prevalence of $19 \%$ for membership of the "high risk" cohort in the 8th month of pregnancy. A random sample of 110 women who scored 7 or less formed the "low risk" cohort.

COMPARISON OF HIGH RISK AND LOW RISK GROUPS

The high risk women were older than low risk women (mean age 25.3 years, SD $6.8 v 23.5$, SD $5.7 ; t=1.9, \mathrm{df}=204, \mathrm{p}<0.05)$. They were more likely to have three or more children, to have had a history of miscarriages in the past, to describe a poorer relationship with their husband or cohabitee, to have a mother living in the rural areas and to describe current health problems (table 1). Half the women in both groups were unemployed and over $90 \%$ married or cohabiting.

OUTCOME OF HIGH RISK AND LOW RISK GROUPS Of the 95 high risk women $44(46 \%)$ became cases and of the 110 low risk women $10(9 \%)$ became cases (OR 8.9, 3.9, 20.8, p<0.0001). This association was even stronger after adjustment for age, marital status, and occupation 
(OR 10.6, 4.8, 23.9, p<0.0001). The predictive accuracy of the SSQ was: sensitivity $81.5 \%$; specificity $66 \%$; positive predictive value $46 \%$; negative predictive value $91 \%$. The CISR domains showed that anxiety, worry, depression, fatigue, and sleep problems were the commonest symptoms being reported by over $90 \%$ of cases.

Variables predicting a poor outcome were examined for the two groups separately. For the high risk group, only the total SSQ score predicted a poor outcome (adjusted OR 1.3, $0.99,1.7, \mathrm{p}=0.05)$. If the high risk cohort was categorised into two groups based on the SSQ score being in the upper tertile range $(=>11)$ or below this score, the former was strongly associated with a poorer outcome (adjusted OR $2.9,1.2,7.5, \mathrm{p}=0.02)$. None of the variables predicted poor outcome for the low risk group.

Obstetric complications were rare; one woman needed a vacuum extraction while another had a caesarean delivery. Both were originally in the low risk group, and the second woman became a case postnatally. One neonatal death was recorded in a woman who was in the high risk group and was a postnatal case. Because of this low frequency of complications, it was not possible to examine their association with postnatal caseness.

A weighted prevalence for postnatal psychiatric disorder was estimated at $16 \%$ taking into account the sampling fraction of the low risk cohort. Although the version of the CISR used in this study does not lead to ICD diagnoses of specific psychiatric disorders, $85 \%$ of the women with a postnatal mental disorder scored high $(=>2)$ on the depression and depressive ideas domains suggesting that postnatal depression was the commonest clinical presentation.

\section{Discussion}

Postnatal mental disorders are a poorly recognised and potentially disabling disorder associated with childbirth in developing countries. One reason for low recognition is the volume of work entailed in searching for a possible mental disorder in postnatal women, particularly when the emphasis on care is on the infants physical well being and immunisation. Discussions with traditional birth attendants and midwives in Harare, Zimbabwe, suggested that if a "high risk" group of women in the antenatal period could be identified, they could reasonably consider increasing the time spent with this group to help prevent or treat a mental disorder after childbirth. With this rationale, this study was conducted to determine whether psychological morbidity in late pregnancy as detected by a simple screening questionnaire could identify women at risk of suffering a postnatal mental disorder. We chose to use a symptom checklist compared with a list of putative risk factors for two reasons: firstly, attempts using risk factors have shown moderate or minimal ability to predict postnatal depression ${ }^{3}{ }^{8}$; and secondly, many risk factors such as previous history of depressive disorders and family history of depression were felt to be difficult to elicit reliably given the conceptual problems with the construct of depression in the Shona culture. ${ }^{14}$ This is the first study of this nature from southern Africa and its findings show unequivocally that psychological morbidity is common both in the last trimester of pregnancy and in the early postnatal period, and that a brief screening questionnaire in the eighth month of pregnancy is reasonably accurate in predicting postnatal mental disorder.

Research examining the link between antenatal and postnatal morbidity has led to conflicting findings; while some studies have reported that most postnatal depression is unrelated to antenatal psychological disorders, ${ }^{5}$ others have reported a strong correlation between the two ${ }^{15} 16$; a recent meta analysis has favoured the second association. ${ }^{2}$ While this variation may point to the differing sociocultural factors operating in different settings, it may also reflect differences in study methodologies such as case criteria and sampling procedures. In the study reported in this paper, women with antenatal psychological morbidity were significantly more likely to develop a postnatal mental disorder than women without antenatal morbidity. This morbidity could be detected by a brief questionnaire, which thus identified women in the final stages of pregnancy who were more likely to suffer from a postnatal mental disorder. Targeting interventions at women in late pregnancy with psychological morbidity may have the potential of changing the course of their illness and help prevent its continuation into the postnatal period. ${ }^{10}$ In settings that are very busy or short staffed, the target population may be further reduced by selecting women who are severely ill, for example those scoring in the upper tertile range of SSQ scores, because this group is the one most likely to remain ill in the postnatal period. Antenatal psychological morbidity was self limited for more than half the women who showed no evidence of psychiatric disorder in the postpartum period. The psychological morbidity in these women may be considered as an adjustment reaction to the stress of the forthcoming delivery, which would also be consistent with the finding that this group of women tended to have lower morbidity scores on the SSQ. These findings suggest that psychological morbidity is both common in the period immediately before childbirth, and that for most of these women, it is transient. Those women who have higher morbidity scores in late pregnancy are more likely to have a postnatal mental illness suggesting that the postnatal illness in this group may be simply a continuation of a more severe antenatal illness. Among women who have no antenatal morbidity, only a small proportion go on to develop a postnatal illness de novo.

The chief limitations of the study are that obstetric data may not be entirely reliable because many women were delivered at home by traditional birth attendants. While the low reported incidence of complications may be partly accounted for by this unreliability, the fact that most of the women were community based (compared with a clinic attending population) may suggest that they were 
comparatvely less likely to suffer from pregnancy related diseases and obstetric complications. Thus, it is not possible to clearly state the association between such complications and postnatal mental disorder. Details of medical problems reported by the women were also not obtained and thus, though there is a strong association between such problems and mental disorder, no specific medical problems could be examined. Another limitation is that a categorical approach was not used in classifying postnatal mental disorders into various types. However, the CISR is specifically designed to detect non-psychotic disorders, which form the main bulk of postnatal mental disorders and in this group of disorders, postnatal depression is the single largest diagnostic category. Depressive symptoms were indeed the commonest clinical presentations in women with a postnatal mental disorder.

It is of interest to note that the prevalence of probable psychological morbidity (as measured by the SSQ) was high in the 8th month of pregnancy. This finding is consistent with other African studies ${ }^{467}$ and is in contrast with the assumption that pregnancy is associated with less psychiatric disorder in the African woman because of the belief that child bearing is the ultimate function society bestows on women. The high prevalence of disorder has been ascribed to the breakup of the traditional support networks, resulting from rapid urbanisation and economic difficulties in many African countries. ${ }^{7}$ Furthermore, women may be more worried before childbirth because of concerns about a successful outcome ${ }^{5}$ and this may account for the association of a history of miscarriages and current medical problems with psychological morbidity in this study. Psychological morbidity in the later stages of pregnancy was also associated with marital disharmony, having three or more children at home, and the mother residing in rural areas. The first association is consistent with findings from developed countries ${ }^{2}$ while the last association points to the impact of urban migration on the mental health of childbearing women as the physical distance from their rural homes interferes with the traditional supportive role that mothers played towards their daughters particularly during pregnancy. Thus, approaches to the management and prevention of mental illness associated with childbirth must recognise not only the high prevalence of antenatal morbidity but also the finding that in most of the women with a postnatal mental illness, the illness probably began before childbirth.

It is recommended that women in the third trimester of pregnancy should be screened with the Shona Symptom Questionnaire to identify women suffering from antenatal psychological morbidity who form a high risk group for postnatal mental illness. Future research needs to examine whether the treatment of psychological morbidity in the late stages of pregnancy or early postnatal period can reduce the morbidity in the puerperium. Thus, women who are in the high risk group may benefit from group
KEY POINTS

- Psychological morbidity is common both in the last trimester of pregnancy and in the early postnatal period.

- Psychological morbidity in the later stages of pregnancy is associated with marital disharmony, having three or more children at home, physical illness, and the mother residing in rural areas.

- A brief symptom based questionnaire in the eighth month of pregnancy accurately predicts which women suffer a postnatal mental disorder.

- Greater severity of antenatal psychological morbidity is associated with a higher level of postnatal morbidity.

- Targeting women with high symptom scores in the antenatal period for interventions may offer a cost effective means of reducing postnatal psychiatric morbidity.

discussions, supportive counselling or antidepressants after delivery.

VP was supported by a Beit Medical Fellowship (UK) and IDRC (Canada). We are grateful to Fungisai Gwanzura, Essie Simunyu, Pat Maramba, and the Village Health Workers in Epworth who helped with the field work and interviews. We are grateful to Martin Prince for his comments.

Funding: the study was supported by a grant from German Technical Cooperation (GTZ, Harare).

Conflicts of interest: none.

\section{Appendix}

Data collected from pregnant women

Age

Marital status

Occupation

Education

Accomodation (rented or owned)

Crowding index (household size/number of rooms) ble)

Husbands/cohabitee occupation (if applica-

Relationship with husband/cohabitee (if applicable)

Household income

Number of children

Whether parents are alive

Residence of parents (rural or urban)

Current medical problems

Number of miscarriages

1 Kumar R. Postnatal Mental illness: a transcultural perspective. Soc Psychiatry Psychiatr Epidemiol 1994;29:250-64.

O'Hara M, Swain A. Rates and risk of postpartum depression-a metaanalysis. Int Rev Psychiatry 1996;8:37-
deram 54.

3 Appleby L, Gregoire A, Platz C, et al. Screening women for high risk of postnatal depression. $\mathcal{F}$ Psychosom Res 1994;38: $539-45$

4 Assael MI, Namboze JM, German GA, Bennett FJ. Psychiatric disturbances during pregnancy in a rural group of African women. Soc Sci Med 1972;6:387-95.

5 Cox JL. Postnatal depression: a comparison of African and Scottish women. Soc Psychiatry 1983;18:25-8.

6 Cox JL. Psychiatric morbidity and pregnancy: a controlled study of 263 semi-rural Ugandan women. Br f Psychiatry 1979;134:401-5.

7 Aderibigbe YA, Gureje O, Omigbodun O. Postnatal emotional disorders in Nigerian women. $\mathrm{Br} f$ Psychiatry 1993;163:645-50.

8 Cooper P, Murray L, Hooper R, West A. The development and validation of a predictive index for postpartum depresand validation of a predictive index
sion. Psychol Med 1996;26:627-34.

9 Holden J. The role of health visitors in postnatal depression. Holden J. The role of health visitors
Int Rev Pschiatry 1996; 8:79-86. 
10 Elliott S, Sanjack M, Leverton T. Parents' groups in pregnancy: a preventative intervention for postnatal
depression? In: Gottleib B, ed. Marshalling social support: formats, processes and effects. Beverly Hills, California:Sage, 1988: 87-110.

11 Patel V, Simunyu E, Gwanzura F, et al. The Shona Symptom Questionnaire: the development of an indigenous measure of non-psychotic mental disorder in Harare. Acta Psychiatr Scand 1997;95:469-75.

12 Lewis G, Pelosi A, Araya R, Dunn G. Measuring psychiatric disorder in the community : a standardized assessment for use by lay interviewers. Psychol Med 1992;22:465-86.
13 Patel V, Gwanzura F, Simunyu E, et al. The explanatory models and phenomenology of common mental disorder in

14 Patel V. Recognizing common mental disorders in primary care in African countries: should "mental" be dropped? Lancet 1996;347:742-4.

5 Mills EP, Finchilescu G, Lea SJ. Postnatal depression--an examination of psychosocial factors. $S$ Afr Med F 1995;85: 99-105

16 McGill H, Burrows VL, Holland LA, et al. Postnatal depression: a Christchurch study. $N Z$ Med fF 1995;108 $162-5$. 